\title{
Interfacial effects on the crystallization and surface properties of poly(/-lactic acid) ultrathin films
}

\author{
Akihiro Udagawa $^{1}$, Toshinori Fujie ${ }^{2}$, Yuko Kawamoto ${ }^{2}$, Akihiro Saito ${ }^{2}$, Shinji Takeoka ${ }^{2}$ and Toru Asahi ${ }^{1,2}$ \\ Polymer crystallization affects structural and mechanical properties of polymer thin films. In this study, we focused on the \\ thermal annealing-induced crystallization in semi-crystalline poly(l-lactic acid) (PLLA) ultrathin films (referred as nanosheets) \\ was investigated in terms of interfacial interaction of PLLA with air and substrate. The surface structure of the PLLA \\ nanosheets observed by atomic force microscopy showed that roughness of the air-side surface increased due to crystallization \\ of PLLA under thermal annealing, whereas that of the substrate-side surface changed little. The elastic moduli and the \\ physical adhesiveness of the nanosheets also changed only on the surface of the air side from crystallization, in contrast to the \\ substrate side. The X-ray diffraction studies of the PLLA nanosheets with different thickness showed that the crystalline contents \\ steeply increased below ca. $200 \mathrm{~nm}$. These results indicated that the crystallization was enhanced near the surface of the air \\ side and restricted near that of the substrate side due to the different interfacial association of the polymer chains in the
} nanosheet.

Polymer Journal (2016) 48, 157-161; doi:10.1038/pj.2015.95; published online 4 November 2015

\section{INTRODUCTION}

Thermodynamics of polymer chains geometrically confined in ultrathin films has attracted much interest in the field of polymer science. In recent years, many experimental studies have shown that glass transition temperatures $\left(T_{\mathrm{g}}\right)$ in supported thin films on a substrate deviates from bulk values due to the change in thermal molecular motion of polymer chains. ${ }^{1-10}$ In particular, polystyrene (PS) has been widely used for such studies on the thermal molecular mobility of polymer chains as a representative of amorphous polymers. In those studies, the $T_{\mathrm{g}}$ of PS was found to be depressed with decreasing film thickness $<100 \mathrm{~nm}$ by ellipsometry ${ }^{4}$, measurements of diffusion rate using fluorescent probes, $^{6}$ X-ray and neutron reflectivity measurements, ${ }^{9}$ inelastic neutron scattering measurements ${ }^{11,12}$ and surface viscoelastic microscopy. ${ }^{13,14}$ In fact, Keddie et al. ${ }^{15}$ reported thermodynamic effects of different substrates on the thermodynamics of poly(methyl methacrylate) (PMMA); the $T_{\mathrm{g}}$ values of PMMA on an $\mathrm{Au}$ substrate were reduced with decreasing thickness and those on a $\mathrm{SiO}_{2}$ substrate increased with decreasing thickness. This thickness dependence should be the result of increasing contribution of interfacial interactions to the thermal mobility of molecular chains inside the films as the thickness decreases. If a strong attraction exists between polymer chains and substrates, restriction of the thermal mobility of polymer chains would make the $T_{\mathrm{g}}$ increase over that of the bulk; otherwise the $T_{\mathrm{g}}$ decreases because the surface layer exists, where thermal mobility of polymer chains is more active, as there is weak restriction between the chains and the substrate. ${ }^{16}$ In addition,
Forrest et al. ${ }^{2}$ reported the results for free-standing PS ultrathin films that the $T_{\mathrm{g}}$ value reduced much more pronouncedly from the bulk value than that of PS films supported on substrates. Thus, interfacial effects are important factors for the thermodynamics of polymer chains in the polymer ultrathin films.

Semicrystalline polymers are crystallized under thermal annealing treatment between the $T_{\mathrm{g}}$ and melting temperature $\left(T_{\mathrm{m}}\right)$ : thermal annealing induces crystallization. The degree of crystallinity, crystal growth rate and morphology have been studied using ultravioletvisible and IR spectroscopy, and atomic force microscopy (AFM) on films with various thicknesses to consider the effects of the interface on the crystallization, resulting from molecular motion. ${ }^{17}$ Wang et al. ${ }^{18}$ proposed a three-layer model for the thermal characteristics of a semi-crystalline polymer film on a substrate just like that for amorphous polymer. This model proposed three layers with higher $T_{\mathrm{g}}$, bulk-like $T_{\mathrm{g}}$ and lower $T_{\mathrm{g}}$ are piled up in one type of semi-crystalline polymer thin film from bottom to top. Thus, in conditions of isothermal crystallization, the crystalline nucleation rate is different between these layers; the rate of the top layer is the fastest and that of the bottom layer is the slowest in this model. This model was consistent with their experimental results of AFM observation to understand the lamella orientation of poly(bisphenol A hexane ether) crystal, although structural difference between the internal region and the substrate side were not deeply discussed.

Poly(l-lactic acid) (PLLA) is one of the semi-crystalline polymers used widely because of its high rigidity and biodegradability, as well as

${ }^{1}$ Department of Advanced Science and Engineering, Graduate School of Advanced Science and Engineering, Waseda University, Tokyo, Japan and ${ }^{2}$ Department of Life Science and Medical Bioscience, Graduate School of Advanced Science and Engineering, Waseda University, Tokyo, Japan

Correspondence: Professor T Asahi, Department of Advanced Science and Engineering, Graduate School of Advanced Science and Engineering, Waseda University, 3-4-1 Okubo, Shinjuku-ku, Tokyo 169-8555, Japan.

E-mail tasahi@waseda.jp.

Received 30 April 2015; revised 7 September 2015; accepted 8 September 2015; published online 4 November 2015 
its biocompatibility. ${ }^{19}$ Due to those properties, PLLA is employed in many kinds of biomaterials as a component. We have recently developed PLLA ultrathin films, that is, PLLA nanosheets, for biomedical applications as wound dressing materials. ${ }^{20,21}$ We previously showed that free-standing PLLA nanosheets have high adhesiveness and high flexibility in the amorphous state, and higher stiffness and selective molecular permeability in the semi-crystalline state caused by thermal annealing treatment. ${ }^{22}$ These results suggest that the crystallization of the PLLA would affect the physical properties of the PLLA nanosheets, similar to the previous reports on the photochemical and electrochemical properties of other crystalline polymers such as poly(3-hexylthiophene) and poly(vinylidene fluoride). ${ }^{23,24}$ However, crystallization effects on the interfacial properties of such polymer films have not been investigated yet, particularly regarding the mobility of polymer chains physically restricted by substrate and enhanced by air.

In this study, we focused on the thermal annealing effect on the surface structural and mechanical properties of both the substrate side and the air side of the PLLA nanosheets. Specifically, we fabricated PLLA nanosheets with different crystallinities using thermal annealing treatment. Then, morphology, elastic modulus and physical adhesiveness of the PLLA nanosheets were quantitatively analyzed for both sides. Finally, the interfacial effect on the crystallization of PLLA nanosheets with different thicknesses was investigated by measuring the crystallinity using X-ray diffraction (XRD).

\section{MATERIALS AND METHODS}

Freestanding PLLA $\left(M_{w}=100000 ; T_{\mathrm{g}}=60-65^{\circ} \mathrm{C}\right)$ nanosheets were fabricated by spin coating using a poly(vinyl alcohol) (PVA, $M_{w}=13000-23$ 000) sacrificial layer method (Figure 1). ${ }^{20-22}$ The following five steps were used in fabrication of the film based on our previous report: ${ }^{22}$ (1) an aqueous solution of PVA (1 wt. \%) was spin-coated (Opticoat MS-A150, Mikasa, Tokyo, Japan) at 4000 r.p.m. for $20 \mathrm{~s}$ on silicon wafers with a $200-\mathrm{nm}$ thermal oxide layer, which was cut into an appropriate size (typically $20 \times 20 \mathrm{~mm}$ ) and washed in a mixture of $\mathrm{H}_{2} \mathrm{SO}_{4} / \mathrm{H}_{2} \mathrm{O}_{2}$ (3:1 by volume) for $\sim 10 \mathrm{~min}$ before rinsing with deionized water; (2) a dichloromethane solution of PLLA was spin-coated onto the PVA layer (4000 r.p.m., $20 \mathrm{~s}$ ) and the thickness of PLLA nanosheet was controlled by adjusting the concentration of PLLA solution as shown in Supplementary Figure S1 of the supporting information; (3) the PLLA layered substrate was annealed at two different conditions $\left(80^{\circ} \mathrm{C}\right.$ for $2 \mathrm{~h}$ and $120^{\circ} \mathrm{C}$ for $2 \mathrm{~h}$ ) for crystallization; (4) the resulting substrate was immersed in distilled water to dissolve the PVA sacrificial layer, resulting in the detachment of PLLA nanosheet from the substrate in the water; (5) the free-standing nanosheet on the water was collected by substrates appropriate for following measurements (Supplementary Figure S2). The PLLA nanosheets without annealing treatment were also prepared by omitting step (3) and dried in vacuo for $2 \mathrm{~h}$. The freestanding PLLA nanosheets were collected on Si wafers or glass substrates, which are appropriate for the experiments conducted in this study. To avoid contamination of the sample surface, all the procedures for the fabrication of PLLA nanosheets were conducted in a clean room (class 10000 ).

The surface morphology of PLLA nanosheet was observed using an AFM in tapping mode (MFP-3D-IO-OLY, Asylum Research, Santa Barbara, CA, USA). ${ }^{22} \mathrm{Si}$ tips with a resonance frequency of $\sim 60 \mathrm{kHz}$ and a spring constant of about $2 \mathrm{~N} \mathrm{~m}^{-1}$ were used; the scan rate was $1.0 \mathrm{~Hz}$ with a scanning density of 512 lines per frame. The elastic moduli of the both surfaces of PLLA nanosheets of $60 \mathrm{~nm}$ thickness were measured by force-curve measurements with the same AFM. A silicon cantilever with a conical-shaped tip was used to indent samples. The spring constant of the cantilever was calculated to be $11.61 \mathrm{~N} \mathrm{~m}^{-1}$ by indenting against a mica disc. To extract the elastic modulus of only the surface region of the PLLA nanosheets, the indentation depth was restricted to $\sim 5 \mathrm{~nm}$ and the force curves were fitted to the equation of Hertz model equation using analysis software equipped with AFM.

The physical adhesiveness was measured using a micro scratch tester (CSR-2000, Rhesca, Tokyo, Japan). ${ }^{25}$ A diamond tip with a radius of $15 \mu \mathrm{m}$ was used; and the scanning velocity and the loading increments were $10 \mu \mathrm{m} \mathrm{s}^{-1}$ and $0.83 \mathrm{mN} \mathrm{s}^{-1}$, respectively. To exclude factors except for the interfacial one, the value for each nanosheet in this test was normalized by dividing by the thickness of the nanosheet.

The crystallization of PLLA nanosheets with various thicknesses annealed at three different conditions $\left(80^{\circ} \mathrm{C}, 2 \mathrm{~h} ; 120^{\circ} \mathrm{C}, 2 \mathrm{~h}\right.$; non-annealed) was evaluated using XRD (RINT-TTR, Rigaku, Tokyo, Japan) with monochromatized $\mathrm{Cu}-\mathrm{K} \alpha$ radiation. The generator settings were $50 \mathrm{kV}$ and $200 \mathrm{~mA}$. As shown in Supplementary Figure S3 of the supporting information, non-annealed PLLA nanosheet showed no peaks attributed to the PLLA crystal. In contrast, the annealed PLLA nanosheet showed a peak $\sim 16.5^{\circ}$, attributed to PLLA crystal. ${ }^{26}$ Therefore, PLLA nanosheet was crystallized after thermal annealing, while asspun PLLA nanosheet was almost fully amorphous before thermal annealing. We calculated the peak area attributed to PLLA crystal of nanosheets with various thicknesses annealed at different conditions (Supplementary Figure S4), by fitting the XRD patterns of each sample to the Voigt function (Supplementary Figure S5). ${ }^{27}$ To obtain the relative crystalline content of each nanosheet, the obtained peak areas were divided by the thickness of the nanosheet in each case.

\section{RESULTS AND DISCUSSIONS}

The surface structures of the PLLA nanosheets are shown in Figures $2 \mathrm{a}-\mathrm{d}$. As can be seen in the images, the surface morphology of the substrate side did not change as a result of thermal annealing. In

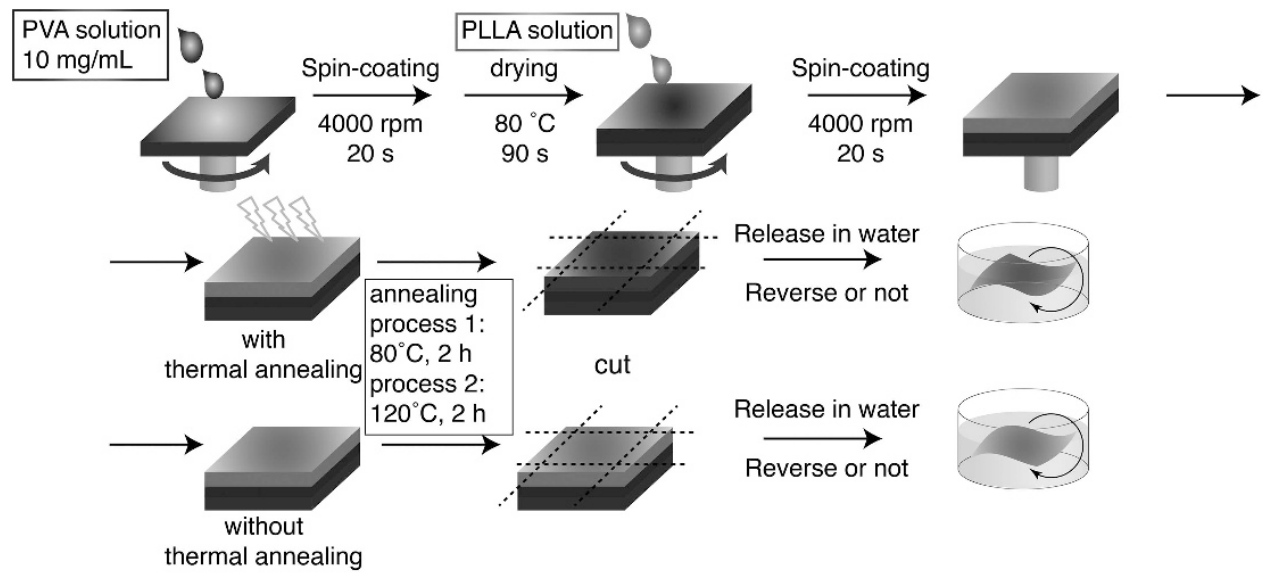

Figure 1 Fabrication method of PLLA nanosheets. PLLA, poly(/-lactic acid); PVA, poly(vinyl alcohol). A full color version of this figure is available at Polymer Journal online. 
contrast, the surface morphology of the air side drastically changed by thermal annealing causing crystallization. Figures $3 \mathrm{a}$ and $\mathrm{b}$ show quantitative analysis of root mean square as the parameter for the surface roughness of the PLLA nanosheets. The root mean square of the air side of the PLLA nanosheets considerably increased as a result of the annealing treatment, and the value annealed at $120^{\circ} \mathrm{C}$ is about 1.5 times as much as that at $80^{\circ} \mathrm{C}$ for each thickness. This is probably caused by differences in the crystallinity of both nanosheets. The substrate-side surface with or without annealing and the air-side surface without annealing exhibit very low root mean square values (less than $\sim 3 \mathrm{~nm}$ ), therefore they are very smooth. By further AFM investigation of the surface morphology of $80^{\circ} \mathrm{C}$ annealed PLLA nanosheet with the additional PVA thin layer, which was spin-coated onto the top of PLLA surface at 4000 r.p.m. for $20 \mathrm{~s}$, it is suggested that there existed little residual PVA on the surface of PLLA nanosheets after being immersed in water. It is also found that the PVA top layer hardly exhibited effect on the crystallization of PLLA ( Supplementary Figure S6b). These results support that the PVA layer on the substrate side of PLLA would be removed with dissolution in water and hardly exhibit effect on the PLLA crystallization.

The elastic moduli of both surfaces of the PLLA nanosheets with 60 -nm thickness are shown in Figure 4. The elastic modulus of the airside surface showed a considerable increase from annealing at $80^{\circ} \mathrm{C}$ (about 1.6 times as high as the value of the sample without annealing), while that of the substrate side showed almost the same between the
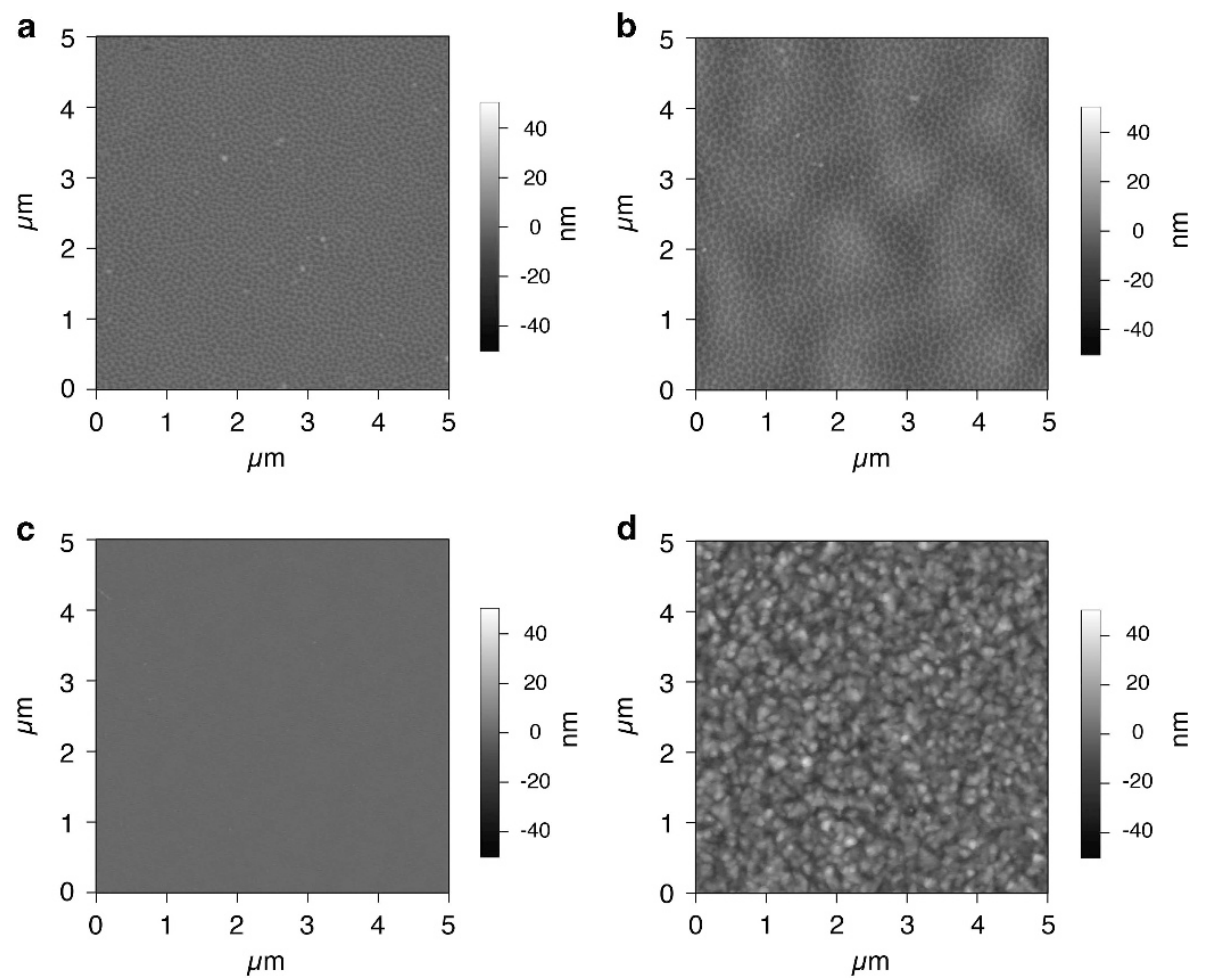

Figure 2 AFM images of PLLA nanosheets (thickness: $60 \mathrm{~nm}$ ): (a and b) substrate-side surface, (c and $\mathbf{d}$ ) air-side surface, (a and $\mathbf{c}$ ) not annealed, and (b and d) annealed at $80^{\circ} \mathrm{C}$ for $2 \mathrm{~h}$. PLLA, poly(/-lactic acid). A full color version of this figure is available at Polymer Journal online.
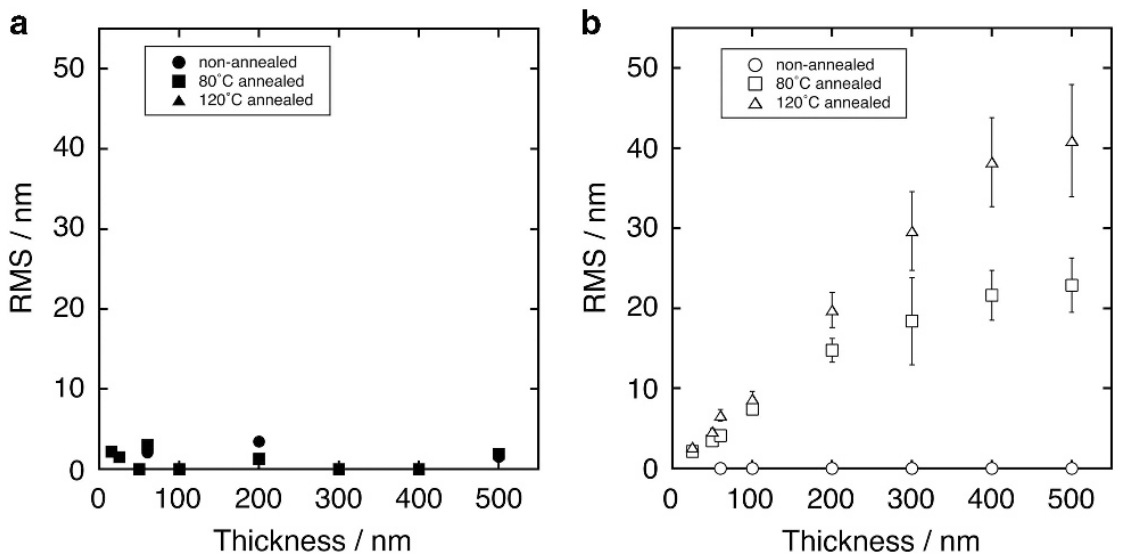

Figure 3 Surface roughness of PLLA nanosheets: (a) substrate side, (b) air side. PLLA, poly(/-lactic acid); RMS, root mean square. 
two annealing conditions (that is, we could not measure the $120^{\circ} \mathrm{C}$ annealed samples because of the difficulty in the measurement). This result indicates that it should be preferable for the surface of the air side to generate crystallites in contrast to the substrate side not to generate crystallites at the same temperature due to the restriction of the polymer chains at the interface. Considering the bulk $T_{\mathrm{g}}\left(\sim 65^{\circ} \mathrm{C}\right)$ of PLLA, it can be proposed that the annealing temperature $\left(80^{\circ} \mathrm{C}\right)$ is above the $T_{\mathrm{g}}$ of the air side and below the $T_{\mathrm{g}}$ of the substrate side.

The results of micro scratch testing of the PLLA nanosheets are shown in Figures $5 \mathrm{a}$ and $\mathrm{b}$. The physical adhesiveness of the substrate side surface of PLLA nanosheets shows the same thickness dependence despite of thermal annealing. However, that of the air side surface surprisingly shows an apparent difference between annealed and nonannealed samples. In particular, only the surface of air side of the PLLA nanosheets lost its adhesiveness by annealing-induced crystallization. Considering the results of morphological observation (Figures 2 and 3), it is suggested that the increased surface roughness has an effect on adhesiveness. These results also agree qualitatively with previous works by Peng et al. ${ }^{28}$

We obtained the XRD patterns of PLLA nanosheets of various thicknesses annealed at different conditions. From the XRD patterns,

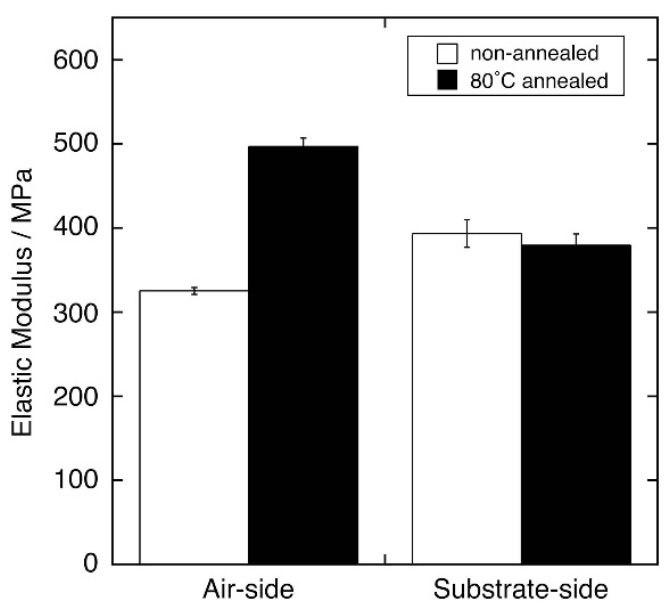

Figure 4 Elastic modulus of PLLA nanosheets (thickness: $60 \mathrm{~nm}$ ). PLLA, poly(/-lactic acid). we calculated the peak area attributed to PLLA crystal by fitting to the Voigt function. Figure 6 shows the relative crystalline contents of the PLLA nanosheets annealed under two different conditions: peak area obtained using XRD divided by its thickness. PLLA nanosheets annealed at both conditions showed the same thickness dependence, such that the crystalline content increases as the thickness decreases from $c a .200 \mathrm{~nm}$, although the contents of both nanosheets seem to be constant for cases thicker than $200 \mathrm{~nm}$. To discuss this critical thickness, interactions with the substrate and the air should be considered. In the case of PLLA nanosheets thicker than $200 \mathrm{~nm}$, three different thermal mobility layers would be piled up: a low mobility layer, a bulk layer and a high mobility layer, from substrate to air. On the other hand, it is inferred that the bulk layer becomes very thin or vanishes in the case of PLLA nanosheets thinner than $200 \mathrm{~nm}$. If the effects of the bulk region on the crystallization become smaller, the residual two layers should become predominant. In fact, in the case of the PLLA nanosheets, crystallization becomes more likely to occur as the thickness decreases from $200 \mathrm{~nm}$, therefore the air-side layer should be predominant for the crystallization. Such predominance should depend on the affinity of the polymer chains and the substrate. ${ }^{15,29,30}$ We also analyzed the difference in the average size of crystallites grown in the PLLA nanosheet annealed at different

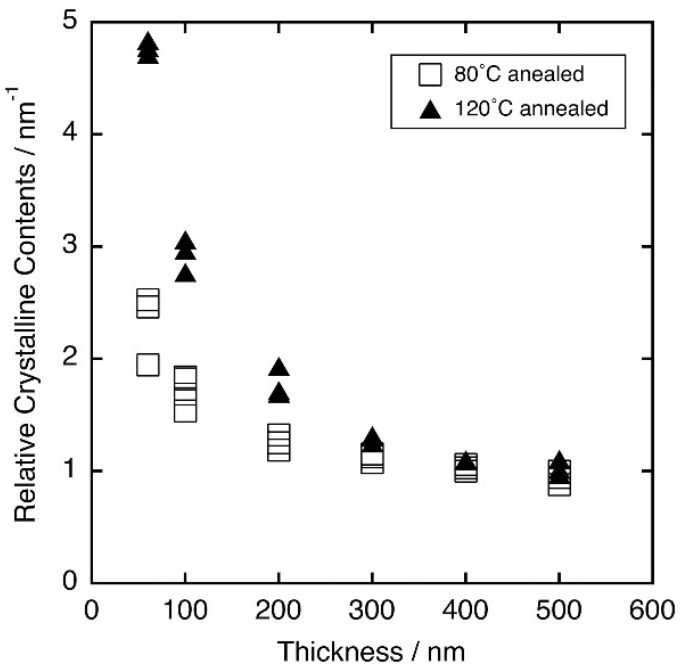

Figure 6 Relative crystalline contents of PLLA nanosheets annealed at two conditions. PLLA, poly(/-lactic acid).
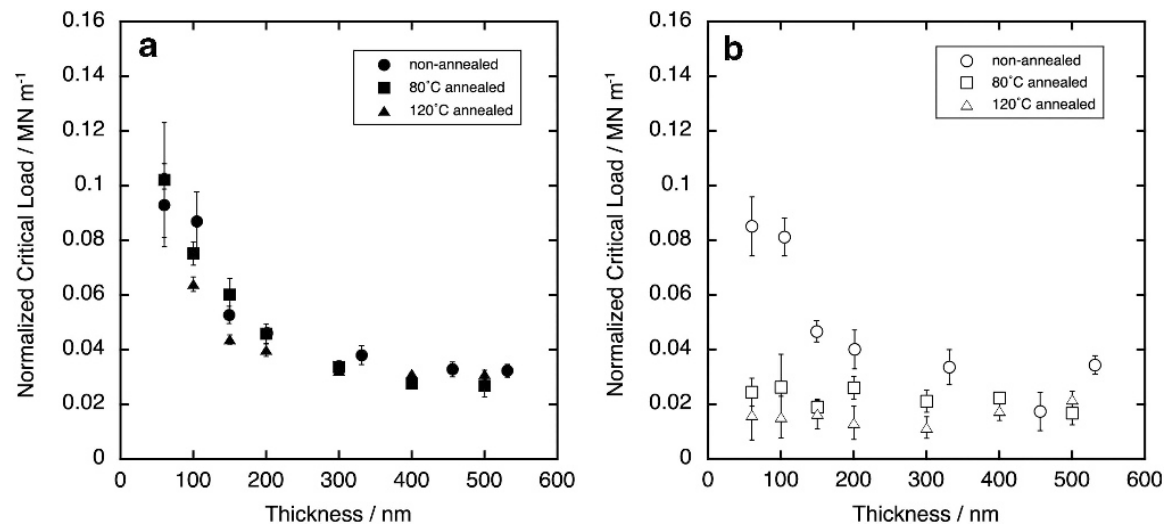

Figure 5 The results of micro scratch test of PLLA nanosheets: (a) substrate-side surface and (b) air-side surface. PLLA, poly(/-lactic acid). 
conditions, by using the full width at half maximum of the diffraction peak as a parameter of Scherrer's equation. However, no significant dependence in the thickness was apparent. These results mean that crystalline size was not changed in the PLLA nanosheets with thickness $<200 \mathrm{~nm}$, although the crystalline contents in the whole PLLA nanosheets increased. This suggests that primary nucleation of PLLA is enhanced in the higher-mobility area, resulting in an increased number of crystals, in spite of the unchanged size of crystallites.

\section{CONCLUSION}

We investigated the interfacial effects on the structural and mechanical properties of both the substrate-side and the air-side surfaces of PLLA nanosheets by thermal annealing treatment. We confirmed that the crystallinity of the PLLA nanosheets was dependent on the annealing temperature. We found that the surface structure and the elastic modulus of the crystallized PLLA nanosheets were different between the air-side and the substrate-side surfaces because the crystalline region is mainly present around the air side. In fact, the adhesiveness of the PLLA nanosheets decreased only on the air-side surface by crystallization under thermal annealing treatment; therefore, we assumed that crystallization near the surface of the substrate side may be restricted by the substrate. Using XRD study, we also found that the crystalline contents increased with decreasing film thickness $<200 \mathrm{~nm}$. Hence, the thermal annealing-induced crystallization results in the different surface properties between air side and substrate side because of the interfacial effects on polymer crystallization. More detailed studies from the viewpoint of internal microstructure may be necessary to understand the crystallization of semi-crystalline polymer in ultrathin films such as PLLA nanosheets in this study. In this regard, high-resolution experimental techniques for non-invasive observation of microstructure such as neutron reflectivity measurements or glazing incident small angle $\mathrm{X}$-ray scattering measurements with a synchrotron radiation beam will clarify the causes of the results obtained in this study.

\section{CONFLICT OF INTEREST}

The authors declare no conflict of interest.

\section{ACKNOWLEDGEMENTS}

We acknowledge funding by the Global COE program for Practical Chemical Wisdom, the project for High-Tech Research Center and the 'Leading Graduate Program in Science and Engineering, Waseda University' from MEXT, Japan. And this work was supported in part by JSPS KAKENHI (Grant Number 15H05355 for TF and 25289252 for ST) from MEXT, Japan and Mizuho Foundation for the Promotion of Science for TF.

1 Keddie, J. L., Jones, R. \& Cory, R. A. Size-dependent depression of the glass transition temperature in polymer films. Europhys. Lett. 27, 59 (1994).

2 Forrest, J., Dalnoki-Veress, K., Stevens, J. \& Dutcher, J. Effect of free surfaces on the glass transition temperature of thin polymer films. Phys. Rev. Lett. 77, 2002-2005 (1996).

3 Forrest, J., Dalnoki-Veress, K. \& Dutcher, J. Interface and chain confinement effects on the glass transition temperature of thin polymer films. Phys. Rev. E 56, 5705-5716 (1997).

4 Kawana, S. \& Jones, R. Character of the glass transition in thin supported polymer films. Phys. Rev. E 63, 021501 (2001).
5 Fukao, K. \& Miyamoto, Y. Glass transitions and dynamics in thin polymer films: dielectric relaxation of thin films of polystyrene. Phys. Rev. E Stat. Phys. 61, 1743-1754 (2000).

6 Ellison, C. J. \& Torkelson, J. M. The distribution of glass-transition temperatures in nanoscopically confined glass formers. Nat. Mater. 2, 695-700 (2003).

7 Ellison, C., Ruszkowski, R., Fredin, N. \& Torkelson, J. Dramatic reduction of the effect of nanoconfinement on the glass transition of polymer films via addition of smallmolecule diluent. Phys. Rev. Lett. 92, 095702 (2004).

8 Tsui, O. K. C., Russell, T. P. \& Hawker, C. J. effect of interfacial interactions on the glass transition of polymer thin films. Macromolecules $\mathbf{3 4}$ 5535-5539 (2001).

9 Kanaya, T., Miyazaki, T., Watanabe, H., Nishida, K., Yamano, H., Tasaki, S. \& Bucknall, D. B. Annealing effects on thickness of polystyrene thin films as studied by neutron reflectivity. Polymer 44, 3769-3773 (2003).

10 Kanaya, T., Inoue, R., Kawashima, K., Miyazaki, T., Tsukushi, I., Shibata, K., Matsuba, G., Nishida, K. \& Hino, M. Glassy dynamics and heterogeneity of polymer thin films. J. Phys. Soc. Jpn. 78, 041004 (2009).

11 Inoue, R., Kanaya, T., Nishida, K., Tsukushi, I. \& Shibata, K. Inelastic neutron scattering study of low energy excitations in polymer thin films. Phys. Rev. Lett. 95, 056102 (2005)

12 Inoue, R., Kanaya, T., Nishida, K., Tsukushi, I., Telling, M., Gabrys, B., Tyagi, M., Soles, C. \& Wu, W. L. Glass transition and molecular mobility in polymer thin films. Phys. Rev. E Stat. Nonlin. Soft Matter Phys. 80, 031802 (2009).

13 Tisato, K., Keiji, T. \& Atsushi, T. Study of the surface glass transition behaviour of amorphous polymer film by scanning-force microscopy and surface spectroscopy. Polymer 39, 4665-4673 (1998).

14 Satomi, N., Takahara, A. \& Kajiyama, T. Determination of surface glass transition temperature of monodisperse polystyrene based on temperature-dependent scanning viscoelasticity microscopy. Macromolecules 32, 4474-4476 (1999).

15 Keddie, J. L., Richard, A. L. \& Cory, R. A. Interface and surface effects on the glass-transition temperature in thin polymer films. Farad. Discuss. 98 219-230 (1994).

16 Tanaka, K., Tateishi, Y., Okada, Y. \& Nagamura, T. Interfacial mobility of polymers on inorganic solids. J. Phys. Chem. B 113, 4571-4577 (2009).

17 Despotopoulou, M. M., Miller, R. D., Rabolt, J. F. \& Frank, C. W. Polymer chain organization and orientation in ultrathin films: a spectroscopic investigation. J. Polym. Sci. B Polym. Phys. 34, 2335-2349 (1998).

18 Wang, Y., Chan, C.-M., Ng, K.-M. \& Li, L. What controls the lamellar orientation at the surface of polymer films during crystallization? Macromolecules 41, 2548-2553 (2008).

19 Garlotta, D. A literature review of poly (lactic acid). J. Polym. Environ. 9, 63-84 (2001).

20 Okamura, Y., Kabata, K., Kinoshita, M., Saitoh, D. \& Takeoka, S. Free-standing biodegradable poly(lactic acid) nanosheet for sealing operations in surgery. Adv. Mater. 21, 4388-4392 (2009).

21 Miyazaki, H., Kinoshita, M., Saito, A., Fujie, T., Kabata, K., Hara, E., Ono, S., Takeoka, S. \& Saitoh, D. An ultrathin poly(L-lactic acid) nanosheet as a burn wound dressing for protection against bacterial infection. Wound Repair Regen. 20, 573-579 (2012).

22 Fujie, T., Kawamoto, Y., Haniuda, H., Saito, A., Kabata, K., Honda, Y., Ohmori, E., Asahi, T. \& Takeoka, S. Selective molecular permeability induced by glass transition dynamics of semicrystalline polymer ultrathin films. Macromolecules 46, 395-402 (2013).

23 Zhokhavets, U., Erb, T., Gobsch, G., Al-Ibrahim, M. \& Ambacher, O. Relation between absorption and crystallinity of poly(3-hexylthiophene)/fullerene films for plastic solar cells. Chem. Phys. Lett. 418, 347-350 (2006).

24 Zhang, Q. M., Xu, H., Fang, F., Cheng, Z. Y., Xia, F. \& You, H. Critical thickness of crystallization and discontinuous change in ferroelectric behavior with thickness in ferroelectric polymer thin films. J. Appl. Phys. 89, 2613 (2001).

25 Rats, D., Hajek, V. \& Martinu, L. Micro-scratch analysis and mechanical properties of plasma-deposited silicon-based coatings on polymer substrates. Thin Solid Films $\mathbf{3 4 0}$, 33-39 (1999).

26 Kalb, B. \& Pennings, A. J. General crystallization behaviour of poly (L-lactic acid). Polymer 21, 607-612 (1980)

27 Langford, J. I. A rapid method for analysing the breadths of diffraction and spectral lines using the Voigt function. J. Appl. Crystallogr. 11, 10-14 (1978).

28 Peng, Z. L. \& Chen, S. H. Effects of surface roughness and film thickness on the adhesion of a bioinspired nanofilm. Phys. Rev. E Stat. Nonlin. Soft Matter Phys. 83, 051915 (2011).

29 Yang, Z., Fujii, Y., Lee, F. K., Lam, C. H. \& Tsui, O. K. C. Glass transition dynamics and surface layer mobility in unentangled polystyrene films. Science $\mathbf{3 2 8}$, 1676-1679 (2010).

30 Paeng, K., Swallen, S. F. \& Ediger, M. D. Direct measurement of molecular motion in freestanding polystyrene thin films. J. Am. Chem. Soc. 133, 8444-8447 (2011).

Supplementary Information accompanies the paper on Polymer Journal website (http://www.nature.com/pj) 